\title{
Diffusiophoresis of a colloidal sphere in nonelectrolyte gradients in a circular cylindrical pore
}

\author{
Huan J. Keh*, Yun S. Hsu \\ Department of Chemical Engineering, National Taiwan University, Taipei 10617, Taiwan, ROC
}

Received 23 August 2005; received in revised form 29 November 2005; accepted 19 December 2005

Available online 17 February 2006

\begin{abstract}
The problem of the diffusiophoretic motion of a spherical particle in a fluid solution of a nonionic solute along the centerline of a circular cylindrical pore is studied theoretically in the quasisteady limit of negligible Reynolds and Peclet numbers. The imposed solute concentration gradient is uniform and parallel to the pore wall, which may be either impermeable to the solute molecules or prescribed with the far-field concentration distribution. The particle-solute interaction layer at the particle surface is assumed to be thin relative to the particle radius and to the particle-wall gap width, but the polarization effect of the diffuse solute in the thin interfacial layer caused by the strong adsorption of the solute is incorporated. The presence of the pore wall causes two basic effects on the particle velocity: first, the local solute concentration gradients on the particle surface are altered by the wall, thereby speeding up or slowing down the particle; secondly, the wall increases viscous retardation of the moving particle. To solve the equations of conservation of mass and momentum, the general solutions are constructed from the fundamental solutions in both cylindrical and spherical coordinates. The boundary conditions are enforced first at the pore wall by the Fourier transforms and then on the particle surface by a collocation technique. Numerical results for the diffusiophoretic velocity of the particle relative to that under identical conditions in an unbounded fluid solution are presented for various values of the relaxation parameter of the particle as well as the relative separation distance between the particle and the pore wall. The collocation results agree well with the approximate analytical solution obtained by using a method of reflections. The wall-corrected particle velocity depends on the surface properties of the particle, the ratio of particle-to-pore radii, and the solutal boundary condition at the wall. In general, the boundary effect on diffusiophoresis is quite significant and complicated.
\end{abstract}

(C) 2006 Elsevier Ltd. All rights reserved.

Keywords: Diffusiophoresis; Colloidal phenomena; Fluid mechanics; Diffusion; Boundary effects; Electrophoresis

\section{Introduction}

Colloidal particles can be driven to move by the application of a nonuniform solute concentration field that interacts with the surface of each particle. This phenomenon, known as diffusiophoresis (Dukhin and Derjaguin, 1974), has been demonstrated experimentally for both charged (Ebel et al., 1988) and uncharged (Staffeld and Quinn, 1989) solutes. In a solution of nonionic solute with constant concentration gradient $\nabla C_{\infty}$, the diffusiophoretic velocity of a particle is (Anderson et al., 1982)

$\mathbf{U}^{(0)}=\frac{k T}{\eta} L^{*} K \nabla C_{\infty}$

\footnotetext{
* Corresponding author. Fax: +886223623040.

E-mail address: huan@ntu.edu.tw (H.J. Keh).
}

and there is no rotational motion of the particle. In Eq. (1), $L^{*}$ is a characteristic length for the particle-solute interaction (of order $1-10 \mathrm{~nm}), K$ is the Gibbs adsorption length characterizing the strength of the adsorption of the molecular solute $[K$ and $L^{*}$ are defined later by Eqs. (6b) and (6c)], $\eta$ is the fluid viscosity, $k$ is Boltzmann's constant, and $T$ is the absolute temperature. Eq. (1) can be applied to an isolated rigid particle of arbitrary shape and size. However, its validity is based on the assumption that the local radii of curvature of the particle are much larger than the thickness of the particle-solute interaction layer (diffuse layer) at the particle surface (of the same order as $L^{*}$ ) and the effect of the polarization of the diffuse solute (the solute continually adsorbs at the upstream edge and desorbs at the downstream edge) in the interfacial layer surrounding the particle is negligible.

An important advance has been made by Anderson and Prieve (1991) in the evaluation of the effect of diffuse-layer 
polarization on diffusiophoresis. They analyzed the diffusiophoretic motion of a colloidal sphere of radius $a$ with a thin but polarized diffuse layer when the suspending nonelectrolyte solution is only slightly nonuniform in solute concentration on the length scale of $a\left(a\left|\nabla C_{\infty}\right| \ll C_{\infty}\right)$ and obtained for the diffusiophoretic velocity of the particle as

$\mathbf{U}_{0}=A \nabla C_{\infty}$,

where the particle's mobility

$A=\frac{k T}{\eta} L^{*} K\left(1+\frac{\beta}{a}\right)^{-1}$

and the definition of the length $\beta$ is given by Eq. (5). For a strongly adsorbing solute (e.g. a surfactant), the relaxation parameter $\beta / a$ (or $K / a$ ) can be much greater than unity. If all the adsorbed solute were "stuck" to the surface of the particle (the diffuseness of the adsorption layer disappears), then $L^{*}=0$ and there would be no diffusiophoretic migration of the particle. In the limit of $\beta / a \rightarrow 0$ (very weak adsorption), the polarization of the diffuse solute in the interfacial layer vanishes and Eq. (2) reduces to Eq. (1). It can be seen from Eqs. (1) and (2) that the effect of polarization of the diffuse layer is to lower the diffusiophoretic mobility of the particle. The reason for this consequence is that the transport of the solute inside the particle-solute interaction layer reduces the concentration gradient along the particle surface.

Eq. (2) serves only for external fluids that extend to infinity in all directions. In real situations of diffusiophoresis, however, colloidal particles are not isolated and will move in the presence of neighboring boundaries. In the limiting case that Eq. (1) is applicable, the normalized velocity field of the unbounded fluid that is dragged by a particle during diffusiophoresis is the same as for electrophoresis of the dielectric particle with an infinitesimally thin electric double layer (Anderson, 1989); thus, the boundary effects on electrophoresis, which have been investigated extensively in the past and summarized by a review article (Chen and Keh, 1999), can be utilized to interpret those on diffusiophoresis. An important result of these investigations is that the boundary effects on electrophoresis (and on diffusiophoresis) are much weaker than on sedimentation, because the disturbance to the fluid velocity field caused by a phoretic particle decays faster than that caused by a Stokeslet.

When the polarization effect of solute species in the diffuse layer surrounding the particle is considered, the boundary effects on diffusiophoresis can be quite different from those on electrophoresis, due to the fact that the particle size and some other unique factors are involved in each transport mechanism. Through the use of a boundary collocation technique, the diffusiophoretic (and electrophoretic) motion of a colloidal sphere with a thin but polarized diffuse layer in the direction perpendicular to a plane wall was examined (Keh and Jan, 1996). Recently, the motion parallel to two plane walls at an arbitrary position between them was also investigated by using a boundary collocation method (Chen and Keh, 2002). Numerical results of wall-correction to Eq. (2) for the particle velocity were presented for various values of the relative separation distances and the parameter $\beta / a$.

In this paper, our purpose is to obtain exact numerical solutions and approximate analytical solutions for the diffusiophoretic motion of a spherical particle with a thin but polarized diffuse layer in a solution of uncharged solute along the centerline of a long circular cylindrical pore. The pore wall may be either impermeable to the solute molecules or prescribed with the linear far-field solute concentration distribution, but the solute-wall interaction energy is assumed to be negligible. The effects of fluid inertia as well as solute convection are ignored. For the case of a particle with a small value of $\beta / a$ undergoing diffusiophoresis near an impermeable pore wall or of a particle with a large value of $\beta / a$ undergoing diffusiophoresis near a pore wall prescribed with the far-field concentration distribution, the solute diffusion around the particle will generate larger concentration gradients on the particle surface relative to those in an infinite medium. These gradients enhance the diffusiophoretic velocity, although their action will be retarded by the viscous interaction of the migrating particle with the wall. The effects of the solutal enhancement and of the hydrodynamic retardation both increase as the ratio of particle-to-pore radii increases. A main object of this work is to determine which effect is overriding at various particle-wall gap widths.

\section{Analysis}

We consider the axisymmetric diffusiophoresis of a spherical particle of radius $a$ in a fluid solution of a nonionic solute along the axis of a long circular cylindrical pore of radius $b$, as shown in Fig. 1. Here $(\rho, \phi, z)$ and $(r, \theta, \phi)$ denote the circular cylindrical and spherical coordinate systems, respectively, and the origin of coordinates is chosen at the particle center. A linear solute concentration field $C_{\infty}(z)$ with a uniform gradient $E_{\infty} \mathbf{e}_{z}$ ( $=\nabla C_{\infty}$, where $E_{\infty}$ is taken to be positive for convenience) is imposed in the surrounding fluid far away from the particle, where $\mathbf{e}_{z}$ is the unit vector in the $z$ direction. It is assumed that the layer of interaction between the solute molecules and the particle surface is thin in comparison with the radius of the particle and the spacing between the particle and the pore

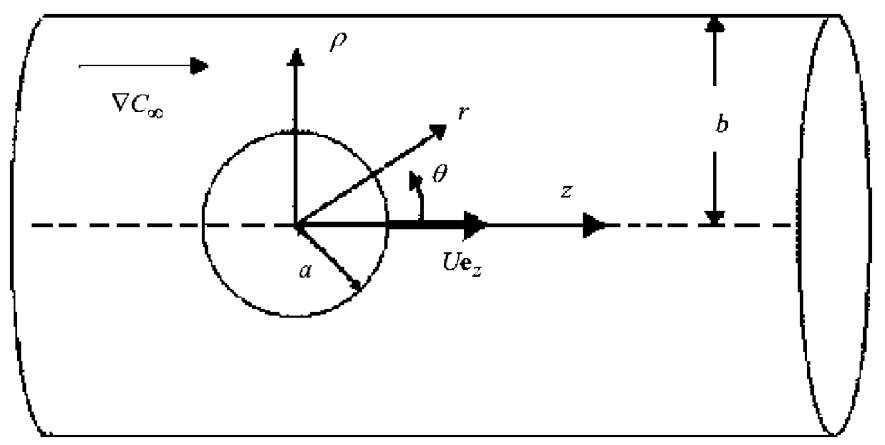

Fig. 1. Geometrical sketch for the diffusiophoresis of a colloidal sphere along the axis of a long circular cylindrical pore. 
wall. Hence, the fluid phase can be divided into two regions: an "inner" region defined as the thin interaction layers adjacent to the solid surfaces and an "outer" region defined as the remainder of the fluid phase. The objective is to obtain the correction to Eq. (2) for the particle velocity due to the presence of the pore.

To determine the diffusiophoretic velocity of the particle, it is necessary to ascertain the concentration and velocity fields in the fluid phase.

\subsection{Solute concentration distribution}

The diffusiophoretic motion of a particle can be considered quasisteady if the Peclet and Reynolds numbers of the system are small. The equation of continuity governing the solute concentration distribution $C$ for the outer region of the fluid solution of constant solute diffusivity is the Laplace equation

$\nabla^{2} C=0$

The above governing equation satisfies the boundary condition at the particle "surface" (outer limit of the thin interfacial layer) obtained by solving for the solute concentration in the inner region and using a matching procedure to ensure a continuous solution in the whole fluid phase (O'Brien, 1983; Anderson and Prieve, 1991),

$r=a: \quad \frac{\partial C}{\partial r}=-\beta \frac{1}{r^{2} \sin \theta} \frac{\partial}{\partial \theta}\left(\sin \theta \frac{\partial C}{\partial \theta}\right)$.

In the above equation, $\beta$ is the relaxation coefficient defined by

$\beta=(1+v P e) K$

where

$P e=\frac{k T}{\eta D} L^{*} K C_{0}$,

$K=\int_{0}^{\infty}\left[\exp \left(-\Phi\left(y_{n}\right) / k T\right)-1\right] \mathrm{d} y_{n}$

$L^{*}=K^{-1} \int_{0}^{\infty} y_{n}\left[\exp \left(-\Phi\left(y_{n}\right) / k T\right)-1\right] \mathrm{d} y_{n}$

and

$v=\left(L^{*} K^{2}\right)^{-1} \int_{0}^{\infty}\left\{\int_{y_{n}}^{\infty}\left[\exp \left(-\Phi\left(y_{n}\right) / k T\right)-1\right] \mathrm{d} y_{n}\right\}^{2} \mathrm{~d} y_{n}$.

In Eq. (6), $\Phi$ represents the potential energy resulting from the interaction between a single solute molecule and the particle surface; $D$ is the solute diffusion coefficient; $y_{n}$ is the normal distance measured from the particle surface into the fluid phase; $C_{0}$ is the bulk concentration of the solute species measured at the particle center in the absence of the particle. To obtain Eqs. (4)-(6), it was assumed that the concentration of solute within the adsorption boundary layer is related to the solute-surface interaction energy by a Boltzmann distribution.
There is no solute to be transferred through the impermeable pore wall and the solute concentration far away from the particle approaches the undisturbed quantities. Thus,

$\rho=b: \quad \frac{\partial C}{\partial \rho}=0$,

$|z| \rightarrow \infty: \quad C \rightarrow C_{\infty}=C_{0}+E_{\infty} z$

Note that we have neglected the relaxation effect in the thin interfacial layer adjacent to the pore wall. For the case of diffusiophoretic motion of a particle in a pore whose wall is prescribed with a linear concentration profile consistent with the far-field solute distribution, Eq. (7) should be replaced by

$\rho=b: \quad C=C_{0}+E_{\infty} z$.

The solute concentration distribution, which is governed by the linear Laplace equation and anti-symmetric with respect to $z$ after the subtraction of $C_{0}$, can be expressed as the superposition

$C=C_{0}+E_{\infty} z+C_{w}+C_{p}$.

Here, $C_{w}$ is a Fourier-Bessel integral solution of Eq. (3) in cylindrical coordinates that represents the disturbance produced by the pore wall and is given by (Keh and Chiou, 1996)

$C_{w}=E_{\infty} \int_{0}^{\infty} R(\omega) I_{0}(\omega \rho) \sin (\omega z) \mathrm{d} \omega$,

where $I_{0}$ is the modified Bessel function of the first kind of order zero and $R(\omega)$ is an unknown function of the separation variable $\omega$. The last term on the right-hand side of Eq. (10), $C_{p}$, is a solution of Eq. (3) in spherical coordinates representing the disturbance generated by the spherical particle and is given by an infinite series in harmonics

$C_{p}=E_{\infty} \sum_{m=1}^{\infty} T_{m} r^{-m-1} P_{m}(\cos \theta) \quad(m$ is odd $)$,

where $P_{m}$ is the Legendre polynomial of order $m$ and $T_{m}$ are unknown constants. Note that a solution for $C$ of the form given by Eqs. (10)-(12) immediately satisfies the boundary condition at infinity in Eq. (8).

Substituting the solute concentration distribution $C$ given by Eqs. (10)-(12) into the boundary condition in Eq. (7) or (9) and applying the Fourier sine transform on the variable $z$ lead to a solution for the function $R(\omega)$ in terms of the coefficients $T_{m}$. After the substitution of this solution into Eqs. (10)-(12) and utilization of the integral representations of the modified Bessel functions, the concentration distribution $C$ can be expressed as

$C=C_{0}+E_{\infty} z+E_{\infty} \sum_{m=1}^{\infty} T_{m} \delta_{m}^{(1)}(r, \theta) \quad(m$ is odd $)$,

where the function $\delta_{m}^{(1)}(r, \theta)$ is defined by Eq. (B.1) in Appendix B. Applying the boundary condition given by Eq. (4) to Eq. (13) yields

$\sum_{m=1}^{\infty} T_{m}\left[\frac{\beta}{a} \delta_{m}^{(3)}(a, \theta)-\delta_{m}^{(2)}(a, \theta)\right]=\left(1-\frac{2 \beta}{a}\right) \cos \theta$, 
where the definitions of functions $\delta_{m}^{(2)}(r, \theta)$ and $\delta_{m}^{(3)}(r, \theta)$ are given by Eqs. (B.2) and (B.3) (which must be performed numerically).

To satisfy the condition in Eq. (14) exactly along the entire surface of the particle would require the solution of the entire infinite array of unknown constants $T_{m}$. However, the collocation method (Leichtberg et al., 1976; Keh and Chiou, 1996) enforces the boundary condition at a finite number of discrete points on the quarter-circular generating arc of the sphere (from $\theta=0$ to $\theta=\pi / 2$, owing to the symmetry of the system geometry and anti-symmetry in the solute concentration field) and truncates the infinite series in Eq. (13) into finite ones. If the spherical boundary is approximated by satisfying the condition of Eq. (4) at $M$ discrete points on its generating arc, the infinite series in Eq. (13) is truncated after $M$ terms, resulting in a system of $M$ simultaneous linear algebraic equations in the truncated form of Eq. (14). This matrix equation can be numerically solved to yield the $M$ unknown constants $T_{m}$ required in the truncated form of Eq. (13) for the solute concentration distribution. The accuracy of the boundary-collocation/truncation technique can be improved to any degree by taking a sufficiently large value of $M$. Naturally, as $M \rightarrow \infty$ the truncation error vanishes and the overall accuracy of the solution depends only on the numerical integration required in evaluating the functions $\delta_{m}^{(2)}$ and $\delta_{m}^{(3)}$ in Eq. (14).

\subsection{Fluid velocity distribution}

Having obtained the solution for the solute concentration distribution on the particle surface which drives the diffusiophoretic migration, we can now proceed to find the flow field. Owing to the low Reynolds number, the fluid motion is governed by the quasisteady fourth-order differential equation for viscous axisymmetric creeping flows

$E^{2}\left(E^{2} \Psi\right)=0$

in which the Stokes stream function $\Psi$ is related to the velocity components in cylindrical coordinates by

$v_{\rho}=\frac{1}{\rho} \frac{\partial \Psi}{\partial z}$,

$v_{z}=-\frac{1}{\rho} \frac{\partial \Psi}{\partial \rho}$

and the Stokes operator $E^{2}$ has the form

$E^{2}=\rho \frac{\partial}{\partial \rho}\left(\frac{1}{\rho} \frac{\partial}{\partial \rho}\right)+\frac{\partial^{2}}{\partial z^{2}}$.

The boundary conditions for the fluid velocity at the particle surface (Anderson and Prieve, 1991), on the pore wall, and far removed from the particle are

$r=a: \quad \mathbf{v}=U \mathbf{e}_{z}-\frac{k T}{\eta} L^{*} K \frac{1}{r} \frac{\partial C}{\partial \theta} \mathbf{e}_{\theta}$,

$$
\begin{aligned}
& \rho=b: \quad \mathbf{v}=-\frac{k T}{\eta} L_{w}^{*} K_{w} \frac{\partial C}{\partial z} \mathbf{e}_{z}, \\
& |z| \rightarrow \infty: \quad \mathbf{v}=-\frac{k T}{\eta} L_{w}^{*} K_{w} E_{\infty} \mathbf{e}_{z}=v_{\infty} \mathbf{e}_{z} .
\end{aligned}
$$

Here, $K_{w}$ and $L_{w}^{*}$ denote the lengths $K$ and $L^{*}$ defined by Eqs. (6b), (6c), respectively, for the interfacial layer at the pore wall, $U$ is the diffusiophoretic velocity of the particle to be determined, and $\partial C / \partial \theta$ and $\partial C / \partial z$ are obtained from the concentration distribution given by Eq. (13). Note that the diffusioosmotic flow caused by the concentration gradient along the pore wall is allowed in Eqs. (19) and (20).

To solve the flow field, we express the stream function, which is symmetric about the plane $z=0$, in the form

$\Psi=-\frac{1}{2} v_{\infty} \rho^{2}+\Psi_{w}+\Psi_{p}$.

Here $\Psi_{w}$ is a solution of Eq. (15) in cylindrical coordinates that represents the disturbance produced by the pore wall and is given by a Fourier-Bessel integral (Leichtberg et al., 1976; Keh and Chiou, 1996)

$\Psi_{w}=\int_{0}^{\infty}\left[X(\omega) \rho I_{1}(\omega \rho)+Y(\omega) \rho^{2} I_{0}(\omega \rho)\right] \cos (\omega z) \mathrm{d} \omega$,

where $X(\omega)$ and $Y(\omega)$ are unknown functions of $\omega$. The second part of $\Psi$, denoted by $\Psi_{p}$, is a solution of Eq. (15) in spherical coordinates representing the disturbance generated by the particle and is given by

$\Psi_{p}=\sum_{n=2}^{\infty}\left(B_{n} r^{-n+1}+D_{n} r^{-n+3}\right) G_{n}^{-1 / 2}(\cos \theta) \quad(n$ is even $)$,

where $G_{n}^{-1 / 2}$ is the Gegenbauer polynomial of the first kind of order $n$ and degree $-1 / 2 ; B_{n}$ and $D_{n}$ are unknown constants. Note that the boundary condition in Eq. (20) is immediately satisfied by a solution of the form given by Eqs. (21)-(23).

Substituting the stream function $\Psi$ given by Eqs. (21)-(23) into the boundary condition (19) and applying the Fourier cosine transform on the variable $z$ lead to a solution for $X(\omega)$ and $Y(\omega)$ in terms of the coefficients $B_{n}$ and $D_{n}$. After the substitution of this solution into Eqs. (21)-(23) and utilization of Eq. (16) and the integral representations of the modified Bessel functions, the fluid velocity components can be expressed as

$$
\begin{aligned}
v_{\rho}= & \sum_{n=2}^{\infty}\left[B_{n} \gamma_{1 n}^{(1)}(r, \theta)+D_{n} \gamma_{2 n}^{(1)}(r, \theta)\right] \\
& -v_{\infty} \sum_{m=1}^{\infty} T_{m} \alpha_{m}^{(1)}(r, \theta), \\
v_{z}= & \sum_{n=2}^{\infty}\left[B_{n} \gamma_{1 n}^{(2)}(r, \theta)+D_{n} \gamma_{2 n}^{(2)}(r, \theta)\right] \\
& -v_{\infty} \sum_{m=1}^{\infty} T_{m} \alpha_{m}^{(2)}(r, \theta),
\end{aligned}
$$

where $n$ is even and the definitions of functions $\gamma_{i n}^{(j)}(r, \theta)$ for $i$ and $j$ equal to 1 or 2 and $\alpha_{m}^{(i)}(r, \theta)$ for $i=1,2$, or 3 are given 
by Eqs. (B.5), (B.6), and (B.13)-(B.15) (which must be performed numerically).

The only boundary condition that remains to be satisfied is that on the particle surface. Substituting Eqs. (13) and (24) into Eq. (18), one obtains

$$
\begin{aligned}
\sum_{n=2}^{\infty}\left[B_{n} \gamma_{1 n}^{(1)}(a, \theta)+D_{n} \gamma_{2 n}^{(1)}(a, \theta)\right] \\
=U_{0}\left(1+\frac{\beta}{a}\right) \sin \theta \cos \theta \\
\quad-\sum_{m=1}^{\infty} T_{m}\left[U_{0}\left(1+\frac{\beta}{a}\right) \alpha_{m}^{(3)}(a, \theta) \cos \theta\right. \\
\left.\quad-v_{\infty} \alpha_{m}^{(1)}(a, \theta)\right] \\
\sum_{n=2}^{\infty}\left[B_{n} \gamma_{1 n}^{(2)}(a, \theta)+D_{n} \gamma_{2 n}^{(2)}(a, \theta)\right] \\
=U-U_{0}\left(1+\frac{\beta}{a}\right) \sin ^{2} \theta \\
\quad+\sum_{m=1}^{\infty} T_{m}\left[U_{0}\left(1+\frac{\beta}{a}\right) \alpha_{m}^{(3)}(a, \theta) \sin \theta\right. \\
\left.\quad+v_{\infty} \alpha_{m}^{(2)}(a, \theta)\right]
\end{aligned}
$$

where $m$ is odd, $n$ is even, and the first $M$ coefficients $T_{m}$ have been determined through the procedure given in the previous subsection.

Eq. (25) can be satisfied by utilizing the collocation technique presented for the solution of the solute concentration field. At the particle surface, Eq. (25) is applied at $N$ discrete points (values of $\theta$ between 0 and $\pi / 2$ ) and the infinite series in Eq. (24) are truncated after $N$ terms. This generates a set of $2 N$ linear algebraic equations for the $2 N$ unknown coefficients $B_{n}$ and $D_{n}$. The fluid velocity field is completely obtained once these coefficients are solved for a sufficiently large value of $N$.

\subsection{Derivation of the particle velocity}

For the fluid flow field given by Eq. (24), the hydrodynamic force acting on the spherical particle can be determined from (Happel and Brenner, 1983)

$$
F=4 \pi \eta D_{2},
$$

where $\eta$ is the fluid viscosity. This expression shows that only the lowest-order coefficient $D_{2}$ contributes to the drag force exerted on the particle by the fluid.

Since the particle is freely suspended in the surrounding fluid, the net force acting on the particle must vanish. Application of this constraint to Eq. (26) gives

$D_{2}=0$.

To determine the diffusiophoretic velocity $U$ of the particle, Eq. (27) and the $2 N$ algebraic equations resulting from Eq. (25) are to be solved simultaneously. When $a / b=0$ (the pore wall is very far from the diffusiophoretic sphere), the result is

$U=U_{0}+v_{\infty}=U_{0}\left[1-\alpha\left(1+\frac{\beta}{a}\right)\right]$,

where $U_{0}$ is the diffusiophoretic velocity of the particle in the absence of the pore wall given by Eq. (2) and the parameter $\alpha=L_{w}^{*} K_{w} / L^{*} K=-v_{\infty} / U_{0}$.

If the particle velocity in Eq. (18) is disabled (i.e., $U=0$ is set), then the force obtained from Eq. (26) can be taken as the diffusiophoretic force exerted on the particle in the cylindrical pore due to the solute concentration gradient $\nabla C_{\infty}$. This force can be expressed as

$F=6 \pi \eta a U_{0} F^{*}$,

where $F^{*}$ is the normalized magnitude of the diffusiophoretic force. The value of $F^{*}$ also equals $f^{*} U / U_{0}$, where $f^{*}$ is the dimensionless Stokes resistance coefficient of the particle (with no slip) migrating along the axis of the cylindrical pore driven by a body force in the absence of the concentration gradient (Leichtberg et al., 1976; Happel and Brenner, 1983) and $U$ is the diffusiophoretic velocity of the particle obtained from Eq. (27).

\section{Results and discussion}

The solution for the diffusiophoretic motion of a spherical particle along the axis of a cylindrical pore, obtained by using the boundary collocation method described in the previous section, is presented in this section. The system of linear algebraic equations to be solved for the coefficients $T_{m}$ is constructed from Eq. (14), while that for $B_{n}$ and $D_{n}$ is composed of Eq. (25). All the numerical integrations to evaluate the functions $\delta_{m}^{(i)}, \gamma_{i n}^{(j)}$, and $\alpha_{m}^{(i)}$ were done by the 180-point Gauss-Laguerre quadrature.

When specifying the points along the quarter-circular generating arc of the spherical particle where the boundary conditions are to be exactly satisfied, the first point that should be chosen is $\theta=\pi / 2$, since this point defines the projected area of the particle normal to the direction of motion and controls the gap between the particle and the pore wall. In addition, the point $\theta=0$ is also important. However, an examination of the systems of linear algebraic equations (14) and (25) shows that the matrix equations become singular if these points are used. To overcome this difficulty, these points are replaced by closely adjacent points, i.e., $\theta=\delta$ and $\pi / 2-\delta$ (Leichtberg et al., 1976). Additional points along the boundary are selected to divide the quarter-circular arc of the particle into equal segments. The optimum value of $\delta$ in this work is found to be $0.1^{\circ}$ (smaller values of $\delta$ would not enhance the accuracy of the solution), with which the numerical results of the particle velocity converge satisfactorily.

Through the use of the same boundary collocation method, Keh and Chiou (1996) obtained numerical solutions for the normalized velocity of a dielectric sphere surrounded by an infinitesimally thin electric double layer undergoing 
Table 1

Normalized diffusiophoretic velocity of a spherical particle along the axis of a circular cylindrical pore computed from the exact boundary-collocation solution and the asymptotic method-of-reflection solution as $\alpha=0$

\begin{tabular}{|c|c|c|c|c|c|c|}
\hline \multirow[t]{3}{*}{$a / b$} & \multicolumn{6}{|l|}{$U / U_{0}$} \\
\hline & \multicolumn{2}{|l|}{$\beta / a=0.1$} & \multicolumn{2}{|l|}{$\beta / a=1$} & \multicolumn{2}{|l|}{$\beta / a=10$} \\
\hline & Exact solution & Asymptotic solution & Exact solution & Asymptotic solution & Exact solution & Asymptotic solution \\
\hline \multicolumn{7}{|c|}{ For an impermeable wall } \\
\hline 0 & 1 & 1 & 1 & 1 & 1 & 1 \\
\hline 0.1 & 0.99851 & 0.99851 & 0.99753 & 0.99753 & 0.99656 & 0.99656 \\
\hline 0.2 & 0.98849 & 0.98850 & 0.98078 & 0.98079 & 0.97319 & 0.97320 \\
\hline 0.3 & 0.96320 & 0.96335 & 0.93801 & 0.93818 & 0.91412 & 0.91441 \\
\hline 0.4 & 0.91871 & 0.92010 & 0.86242 & 0.86393 & 0.81274 & 0.81560 \\
\hline 0.5 & 0.85386 & 0.86151 & 0.75344 & 0.76114 & 0.67454 & 0.69066 \\
\hline 0.6 & 0.76923 & 0.79961 & 0.61644 & 0.64418 & 0.51523 & 0.57798 \\
\hline 0.7 & 0.66520 & 0.76235 & 0.46120 & 0.53925 & 0.35477 & 0.54119 \\
\hline 0.8 & 0.53789 & 0.80512 & 0.29948 & 0.48181 & 0.20908 & 0.65989 \\
\hline 0.9 & 0.36561 & 1.02990 & 0.14265 & 0.50907 & 0.08943 & 1.00470 \\
\hline 0.95 & 0.23484 & & 0.06909 & & 0.04079 & \\
\hline 0.99 & 0.06465 & & 0.01343 & & 0.00751 & \\
\hline 0.995 & 0.03423 & & 0.00669 & & 0.00371 & \\
\hline 0.999 & 0.007 & & 0.001 & & 0.0007 & \\
\hline \multicolumn{7}{|c|}{ For a wall prescribed with the far-field solute concentration profile } \\
\hline 0 & 1 & 1 & 1 & 1 & 1 & 1 \\
\hline 0.1 & 0.99778 & 0.99778 & 0.99803 & 0.99804 & 0.99829 & 0.99829 \\
\hline 0.2 & 0.98273 & 0.98274 & 0.98471 & 0.98472 & 0.98671 & 0.98672 \\
\hline 0.3 & 0.94428 & 0.94445 & 0.95076 & 0.95091 & 0.95731 & 0.95747 \\
\hline 0.4 & 0.87599 & 0.87747 & 0.89038 & 0.89175 & 0.90517 & 0.90656 \\
\hline 0.5 & 0.77631 & 0.78383 & 0.80173 & 0.80887 & 0.82852 & 0.83590 \\
\hline 0.6 & 0.64810 & 0.67524 & 0.68623 & 0.71272 & 0.72783 & 0.75617 \\
\hline 0.7 & 0.49751 & 0.57464 & 0.54718 & 0.62552 & 0.60421 & 0.69143 \\
\hline 0.8 & 0.33250 & 0.51648 & 0.38804 & 0.58493 & 0.45693 & 0.68688 \\
\hline 0.9 & 0.16220 & 0.54513 & 0.20975 & 0.64913 & 0.27767 & 0.82101 \\
\hline 0.95 & 0.07866 & & 0.11141 & & 0.16555 & \\
\hline 0.99 & 0.01500 & & 0.02446 & & 0.04617 & \\
\hline 0.995 & 0.00742 & & 0.01253 & & 0.02584 & \\
\hline 0.999 & 0.001 & & 0.002 & & 0.006 & \\
\hline
\end{tabular}

electrophoresis along the axis of a circular cylindrical pore with an insulating wall. These results, which can apply to the case of diffusiophoresis of a sphere with $\beta / a=0$ (no polarization of the diffuse solute in the thin layer surrounding the particle) along the axis of a circular pore with an impermeable wall, are found to agree excellently with our solutions and are not numerically presented here for conciseness. In this earlier work, the electric field lines (diffusive flux lines) and the equipotential lines (constant concentration lines) around a sphere in an unbounded fluid and the distortion of these contours due to the presence of the pore wall are graphically exhibited, respectively. The potential (concentration) gradients along the surface of a sphere in the pore are larger than the corresponding values of the sphere in an unbounded medium, and the difference increases with an increase in the separation parameter $a / b$. On the other hand, the streamline patterns for an infinite fluid surrounding a sphere undergoing electrophoresis (diffusiophoresis) and for the corresponding motion along the axis of a circular cylindrical pore are also depicted by Anderson (1986) and Keh and Chiou (1996), respectively, and a comparison between them shows that the fluid circulation around the sphere is distorted substantially by the pore wall.
The collocation solutions for the diffusiophoretic velocity of a spherical particle along the axis of a cylindrical pore under the situations of an impermeable wall and a wall with the imposed far-field solute concentration distribution for different values of the relaxation parameter $\beta / a$ and separation parameter $a / b$ are presented in Tables 1 and 2 for the particular cases of $\alpha=0$ (no diffusioosmotic flow at the pore wall) and of $\alpha=1$ (the same diffusioosmotic slip properties at the particle surface and at the pore wall), respectively. The velocity for the diffusiophoretic motion of an identical particle in an infinite fluid, $U_{0}$, given by Eq. (2), is used to normalize the boundary-corrected values. All of the results obtained under the collocation scheme converge satisfactorily to at least the significant figures shown in the table. The accuracy and convergence behavior of the truncation technique is principally a function of the ratio $a / b$. For the most difficult case with $a / b=0.999$, the numbers of collocation points $M=52$ and $N=52$ are sufficiently large to achieve this convergence.

In Appendix A, an approximate analytical solution for the same diffusiophoretic motion as that considered here is also obtained by using a method of reflections. The particle velocity is given by Eq. (A.13), which is a power series expansion 
Table 2

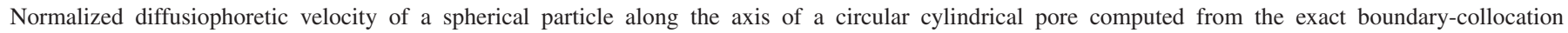
solution and the asymptotic method-of-reflection solution as $\alpha=1$

\begin{tabular}{|c|c|c|c|c|c|c|}
\hline \multirow[t]{3}{*}{$a / b$} & \multicolumn{6}{|l|}{$-U / U_{0}$} \\
\hline & \multicolumn{2}{|l|}{$\beta / a=0.1$} & \multicolumn{2}{|l|}{$\beta / a=1$} & \multicolumn{2}{|l|}{$\beta / a=10$} \\
\hline & Exact solution & Asymptotic solution & Exact solution & Asymptotic solution & Exact solution & Asymptotic solution \\
\hline \multicolumn{7}{|c|}{ For an impermeable wall } \\
\hline 0 & 0.1 & 0.1 & 1 & 1 & 10 & 10 \\
\hline 0.1 & 0.10047 & 0.10047 & 1.00374 & 1.00374 & 10.0276 & 10.0276 \\
\hline 0.2 & 0.10370 & 0.10370 & 1.02891 & 1.02889 & 10.2094 & 10.2093 \\
\hline 0.3 & 0.11214 & 0.11210 & 1.09201 & 1.09171 & 10.6417 & 10.6384 \\
\hline 0.4 & 0.12791 & 0.12752 & 1.20022 & 1.19759 & 11.3089 & 11.2783 \\
\hline 0.5 & 0.15315 & 0.15100 & 1.34913 & 1.33575 & 12.0702 & 11.9135 \\
\hline 0.6 & 0.19106 & 0.18233 & 1.52354 & 1.47569 & 12.7080 & 12.1731 \\
\hline 0.7 & 0.24813 & 0.21901 & 1.70074 & 1.56755 & 13.0114 & 11.6997 \\
\hline 0.8 & 0.34070 & 0.25440 & 1.85503 & 1.54926 & 12.8425 & 10.5591 \\
\hline 0.9 & 0.52148 & 0.27430 & 1.96165 & 1.36360 & 12.1548 & 10.0067 \\
\hline 0.95 & 0.70417 & & 1.99034 & & 11.6266 & \\
\hline 0.99 & 0.98513 & & 1.99963 & & 11.1312 & \\
\hline 0.995 & 1.03878 & & 1.99991 & & 11.0658 & \\
\hline 0.999 & 1.09 & & 2.00 & & 11.01 & \\
\hline \multicolumn{7}{|c|}{ For a wall prescribed with the far-field solute concentration profile } \\
\hline 0 & 0.1 & 0.1 & 1 & 1 & 10 & 10 \\
\hline 0.1 & 0.10120 & 0.10120 & 1.00324 & 1.00324 & 10.0259 & 10.0259 \\
\hline 0.2 & 0.10951 & 0.10950 & 1.02501 & 1.02500 & 10.1984 & 10.1983 \\
\hline 0.3 & 0.13154 & 0.13147 & 1.07967 & 1.07939 & 10.6284 & 10.6222 \\
\hline 0.4 & 0.17309 & 0.17251 & 1.17431 & 1.17180 & 11.3443 & 11.3203 \\
\hline 0.5 & 0.23909 & 0.23612 & 1.30749 & 1.29442 & 12.3160 & 12.1877 \\
\hline 0.6 & 0.33386 & 0.32323 & 1.46998 & 1.42135 & 13.4199 & 12.9272 \\
\hline 0.7 & 0.46160 & 0.43147 & 1.64743 & 1.50256 & 14.4571 & 12.9458 \\
\hline 0.8 & 0.62697 & 0.55469 & 1.81683 & 1.45615 & 15.1255 & 11.1897 \\
\hline 0.9 & 0.83620 & 0.68275 & 1.95533 & 1.15797 & 14.8843 & 5.8856 \\
\hline 0.95 & 0.96023 & & 1.99964 & & 14.0118 & \\
\hline 0.99 & 1.07063 & & 2.00923 & & 12.2838 & \\
\hline 0.995 & 1.08522 & & 2.00655 & & 11.8351 & \\
\hline 0.999 & 1.097 & & 2.0022 & & 11.32 & \\
\hline
\end{tabular}

in $\lambda(=a / b)$. The values of the wall-corrected normalized particle velocity calculated from this asymptotic solution, with the $\mathrm{O}\left(\lambda^{9}\right)$ term neglected, are also listed in Tables 1 and 2 for comparison. It can be seen that the asymptotic formula of Eq. (A.13) from the method of reflections for $U / U_{0}$ agrees very well with the exact results as long as $\lambda \leqslant 0.5$; the errors in all cases are less than $2.4 \%$. However, the accuracy of Eq. (A.13) deteriorates rapidly, as expected, when the relative spacing between the particle and the pore wall becomes small.

The exact numerical solutions for the normalized velocity $U / U_{0}$ of a spherical particle undergoing diffusiophoresis along the axis of a cylindrical pore as a function of $a / b$ for the case of $\alpha=0$ are depicted in Fig. 2 for various values of $\beta / a$. Certainly, the particle moves with the velocity $U_{0}$ given by Eq. (2) as $a / b=0$ and the diffusiophoretic mobility of the particle is a monotonic decreasing function of $a / b$. For the particular case of a particle with $\beta / a=0$ in a pore with an impermeable wall, the diffusiophoretic mobility of the particle is a finite value (about $1 / 2$, a result due to the combined effects of solutal enhancement and hydrodynamic retardation) in the limit of $a / b \rightarrow 1$. For all the other cases, the particle mobility tends to vanish at this limit. The wall-corrected normalized diffusiophoretic mobility $U / U_{0}$ of the particle decreases with an increase in $\beta / a$ for the case of an impermeable wall (the boundary condition (7) is used), but increases with an increase in $\beta / a$ for the case of a pore wall prescribed with the far-field solute concentration distribution (the boundary condition (9) is used), keeping the ratio $a / b$ unchanged. This behavior is expected knowing that the solute concentration gradients on the particle surface near an impermeable wall decrease as the relaxation parameter $\beta / a$ increases and these gradients near a wall with the imposed farfield concentration distribution increase as $\beta / a$ increases (see the analysis in Appendix A). When $\beta / a=1 / 2$, the two types of pore wall will result in the same effects on the diffusiophoretic motion of the particle. In this particular case, the effect of solutal interaction between the particle and the wall disappears, and the relative diffusiophoretic mobility of the particle decreases monotonically with $a / b$ solely owing to the hydrodynamic resistance exerted by the presence of the wall.

For the special situation of $\alpha=1$, the collocation solutions for the normalized diffusiophoretic velocity $U / U_{0}$ of a spherical particle along the axis of a cylindrical pore as a function of 


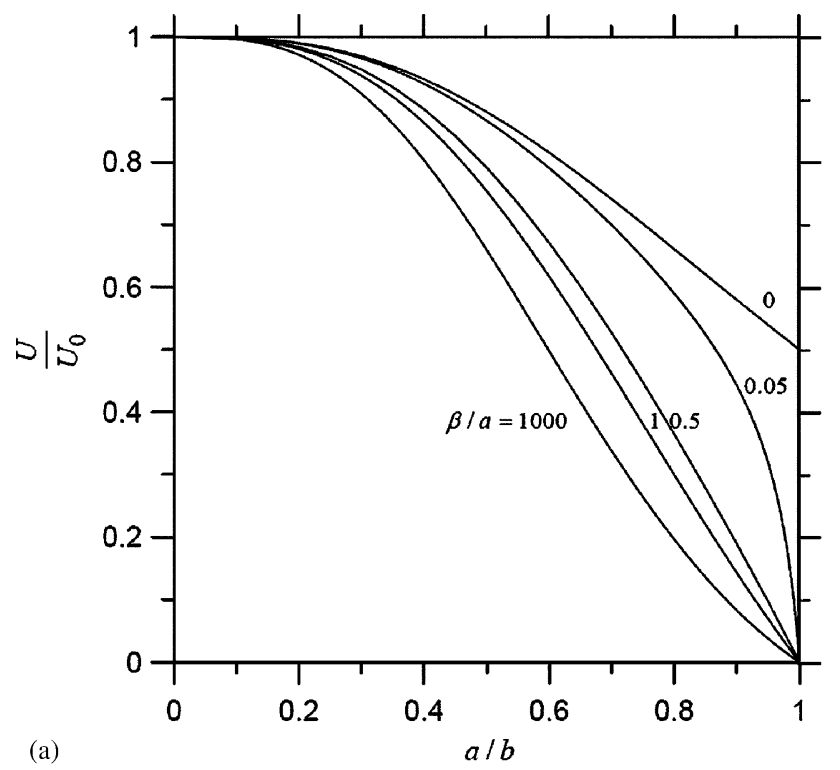

(a)

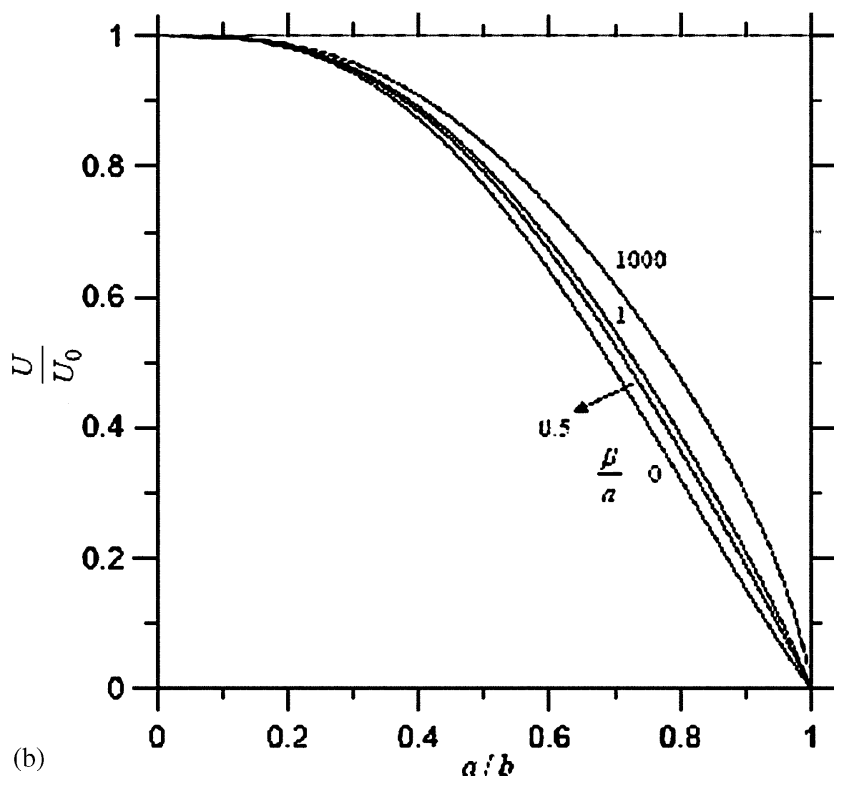

Fig. 2. Plots of the normalized diffusiophoretic velocity of a colloidal sphere along the axis of a circular cylindrical pore with $\alpha=0$ versus the ratio $a / b$ for various values of $\beta / a$ : (a) the case of an impermeable wall; (b) the case of a wall on which the far-field solute concentration profile is imposed.

$a / b$ are shown in Fig. 3 for various values of $\beta / a$. Due to the existence of the uniform diffusioosmotic flow of the fluid far from the particle in the negative $z$ direction caused by the interaction between the imposed solute concentration gradient and the pore wall, the ratio $U / U_{0}$ is no longer equal to unity but equals $-\beta / a$ as required by Eq. (28) when $a / b=0$. When $\beta / a=$ 0 , the velocity of the particle in a pore with an impermeable wall vanishes, irrespective of the value of $a / b$. For any finite value of $\beta / a$ or a pore wall prescribed with the imposed farfield concentration distribution, the bulk-phase diffusioosmotic flow of the fluid dominates and the particle migrates in the direction opposite to that which would occur if it is undergoing diffusiophoresis in an unbounded fluid $\left(U / U_{0}<0\right)$. The value

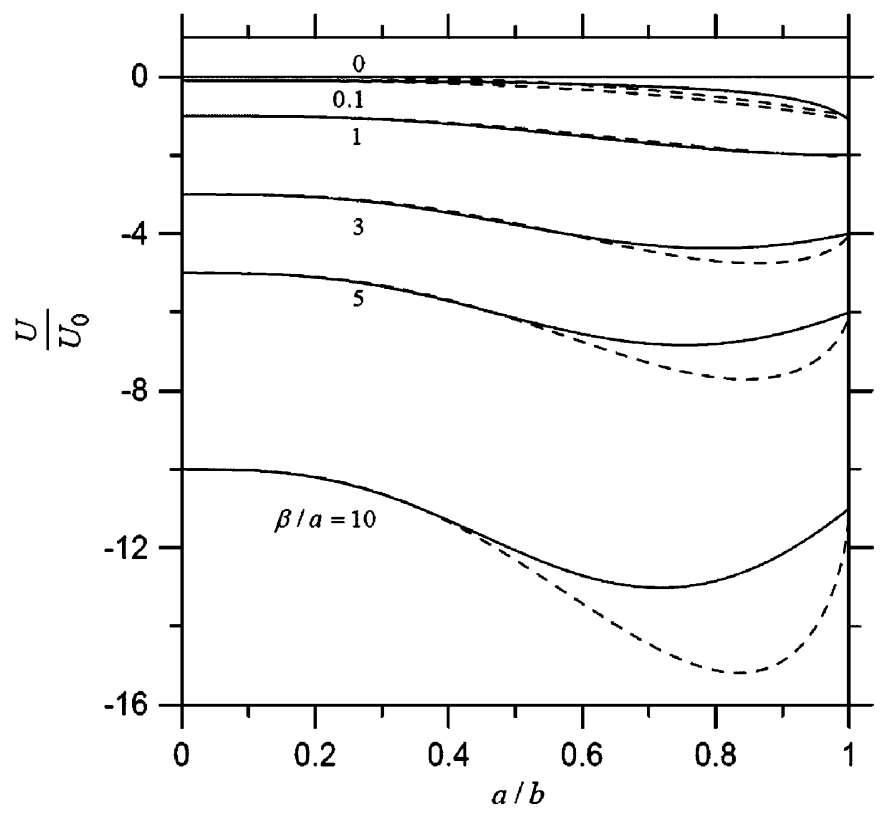

Fig. 3. Plots of the normalized diffusiophoretic velocity of a colloidal sphere along the axis of a circular cylindrical pore with $\alpha=1$ versus the ratio $a / b$ for various values of $\beta / a$. The solid curves represent the case of an impermeable wall, and the dashed curves denote the case of a wall on which the far-field solute concentration profile is imposed.

of $U / U_{0}$ is a monotonic decreasing function of $a / b$ if $\beta / a$ is small (say, less than unity). If $\beta / a$ is large (say, greater than 2 ), the value of $U / U_{0}$ decreases with an increase in $a / b$ as $a / b$ is small, but increases from a minimum with increasing $a / b$ as $a / b$ is sufficiently large. For a given value of $a / b$, the value of $U / U_{0}$ decreases with an increase in $\beta / a$.

In Figs. 4 and 5, plots of the collocation results of $U / U_{0}$ of the diffusiophoretic sphere versus $a / b$ under the situation of $\alpha=0.5$ and 2, respectively, are drawn for various values of $\beta / a$. Again, the bulk-phase diffusioosmotic flow of the fluid in the opposite direction exists and Eq. (28) predicts the limiting case of $a / b=0$. Fig. 4 depicts a typical situation of $0<\alpha<1$ (with the two bounds shown in Figs. 2 and 3). For the particular case of a particle with $\beta / a=0$ in a pore with an impermeable wall, $U / U_{0}$ is always a positive value. Again, the value of $U / U_{0}$ is a monotonic decreasing function of $a / b$ if $\beta / a$ is small and has a minimum at a less-than-unity value of $a / b$ if $\beta / a$ is large. When $\beta / a>\alpha^{-1}-1$, the value of $U / U_{0}$ is always negative. Fig. 5 represents a typical situation of $\alpha>1$ (the effect of the bulkphase diffusioosmotic flow is stronger), in which the value of $U / U_{0}$ is always negative. It shows that, for the case of $\beta / a=0$, the magnitude of the velocity of the particle in a pore with an impermeable wall decreases with an increase in $a / b$.

For the diffusiophoretic motion of a spherical particle of radius $a$ situated at the center of a spherical cavity of radius $b$, the normalized particle mobility can be determined analytically as

$$
\frac{U}{U_{0}}=\frac{2-5 \lambda^{3}+3 \lambda^{5}}{2\left(1+G \lambda^{3}\right)\left(1-\lambda^{5}\right)}+\alpha\left(1+\frac{\beta}{a}\right) \frac{3-5 \lambda^{2}+2 \lambda^{5}}{3\left(1-\lambda^{5}\right)}
$$




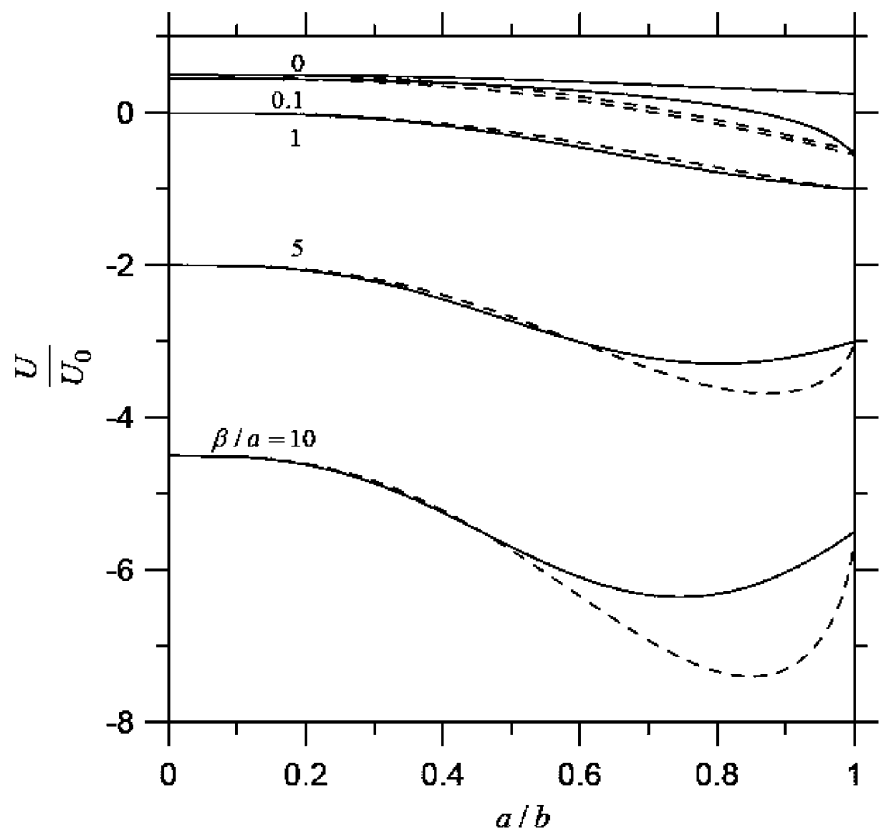

Fig. 4. Plots of the normalized diffusiophoretic velocity of a colloidal sphere along the axis of a circular cylindrical pore with $\alpha=0.5$ versus the ratio $a / b$ for various values of $\beta / a$. The solid curves represent the case of an impermeable wall, and the dashed curves denote the case of a wall on which the far-field solute concentration profile is imposed.

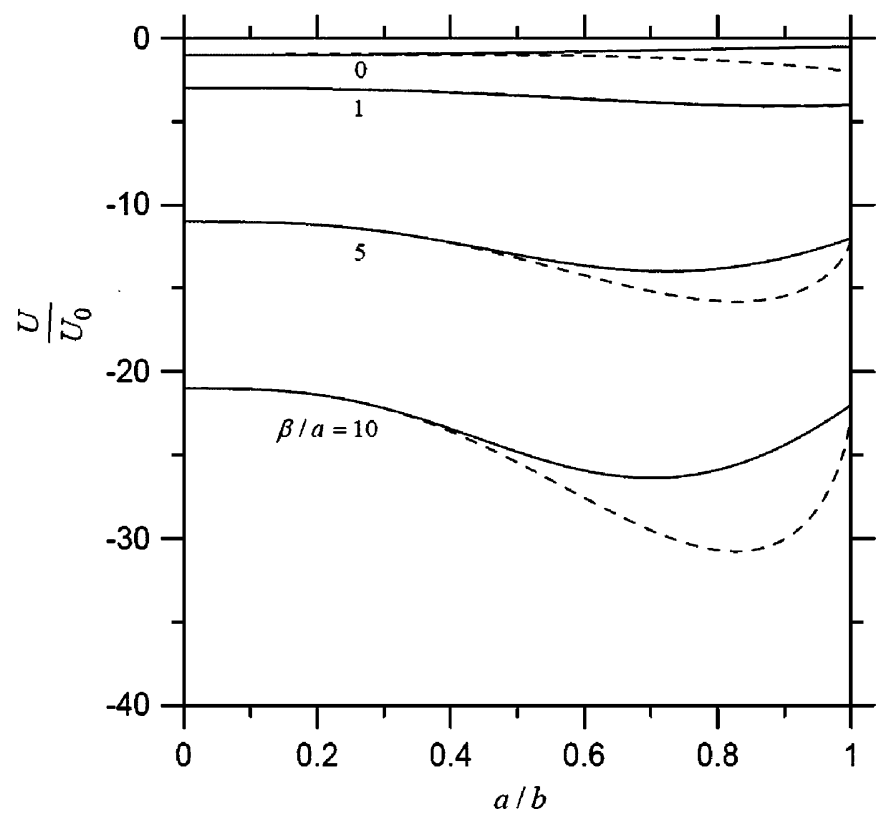

Fig. 5. Plots of the normalized diffusiophoretic velocity of a colloidal sphere along the axis of a circular cylindrical pore with $\alpha=2$ versus the ratio $a / b$ for various values of $\beta / a$. The solid curves represent the case of an impermeable wall, and the dashed curves denote the case of a wall on which the far-field solute concentration profile is imposed.

where

$$
G=\frac{\frac{1}{2}-\frac{\beta}{a}}{1+\frac{\beta}{a}}
$$

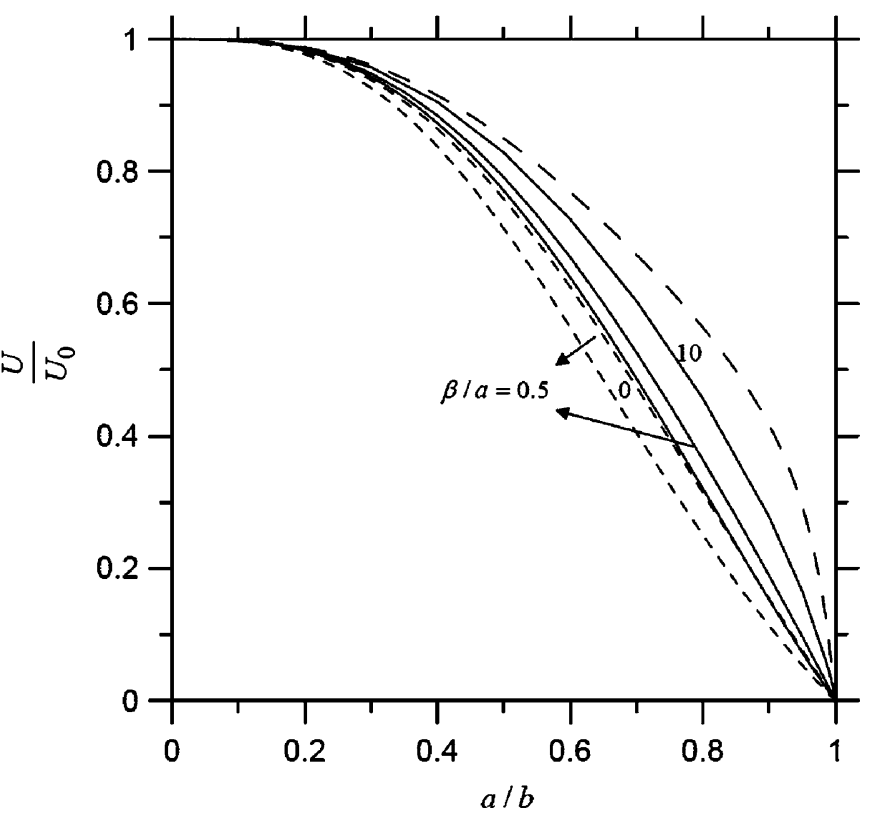

Fig. 6. A comparison of the normalized diffusiophoretic velocities of a colloidal sphere along the axis of a circular cylindrical pore (solid curves) and located at the center of a spherical pore (dashed curves) for various values of $a / b$ and $\beta / a$ (or $G$ ). The solute concentration at the pore wall is taken as the distribution giving rise to the constant gradient $E_{\infty}$ in the absence of the particle.

and $\lambda=a / b$. Obviously, $-1 \leqslant G \leqslant 1 / 2$, with the upper and lower bounds occurring at the limits $\beta / a=0$ and $\beta / a \rightarrow \infty$, respectively. To obtain Eq. (30), the solute concentration at the cavity wall is taken as the distribution giving rise to the constant gradient $E_{\infty}$ in the cavity when the particle does not exist. This normalized diffusiophoretic mobility, which depends on the parameters $\beta / a$ and $\alpha$, decreases monotonically from $1+\alpha(1+\beta / a)$ at $\lambda=0$ to zero as $\lambda \rightarrow 1$. The term involving $\alpha$ in Eq. (30) represents the contribution due to the diffusioosmotic flow of a recirculation pattern in the cavity (the fluid moves in the direction opposite to that of the prescribed solute concentration gradient near the cavity wall at $\theta=\pi / 2$ and in the direction along with the prescribed gradient around the $z$-axis) that arises from the interaction of the prescribed gradient with the slip cavity wall. A comparison of the collocation results for the diffusiophoretic mobility of the particle in a corresponding cylindrical pore (whose wall is prescribed with the far-field solute concentration distribution) with Eq. (30) is presented in Fig. 6 under the situation of $\alpha=0$. It can be seen that the boundary effects on diffusiophoresis in cylindrical and spherical pores are quite similar and the mobility retardation in the cylindrical pore can be greater or smaller than that in the spherical cavity with the same values of $\beta / a$ and $a / b$. The agreement between the numerical values of the two wall-corrected mobilities for the case of $G=0(\beta / a=1 / 2)$ is quite good. The similarity in the boundary effects in these pore geometries reflects the fact that the deformation of solute flux field and the viscous retardation in the cylindrical pore are greatest at the point of closest approach of the particle to the pore wall, 
with the geometry of this region being analogous to that of a sphere in the spherical cavity. Note that the diffusiophoresis of a sphere in a spherical cavity might represent a real system of the chemotactic movement of a tiny particle in a biological cell.

For the creeping motion of a spherical particle on which a constant body force $F \mathbf{e}_{z}$ such as gravity is exerted along the axis of a circular cylindrical pore, the particle velocity was obtained in a power series of $\lambda(=a / b)$ up to $\mathrm{O}\left(\lambda^{10}\right)$ (Happel and Brenner, 1983) and by using the boundary-collocation method (Leichtberg et al., 1976). A comparison of the boundary effects on the translation of the sphere under gravity (in which $U_{0}=$ $F / 6 \pi \eta a)$ and on the diffusiophoresis indicates that the wall effect on diffusiophoretic motion is much weaker than that on a sedimenting particle.

\section{Conclusions}

In this work, the exact numerical solutions and approximate analytical solutions for the quasisteady diffusiophoretic motion of a colloidal sphere along the axis of a circular cylindrical pore have been obtained by using the boundary-collocation technique and the method of reflections, respectively. Both the cases of an impermeable pore wall and of a pore wall with the imposed far-field solute concentration distribution were examined in the limit of vanishingly small Reynolds and Peclet numbers. It has been found that the agreement between the collocation solution and the reflection solution is quite good, and the boundary effect on diffusiophoretic motion of a particle is significant and complicated. The diffusiophoretic mobility of a particle in a pore is generally, but not necessarily, a monotonic decreasing function of the separation parameter $a / b$. When the value of $a / b$ is close to unity, the effect of a lateral wall can speed up or slow down the particle velocity relative to its isolated value depending on the value of the relaxation parameter $\beta / a$ of the particle and the solutal boundary condition at the wall. This behavior reflects the competition between the hydrodynamic retardation exerted by the neighboring wall on the particle migration and the possible diffusiophoretic enhancement due to the solutal interaction between the particle and the lateral wall.

The diffusiophoretic mobility of a spherical particle on the median plane between two parallel plane walls with $\alpha=0$ was calculated in a previous work (Chen and Keh, 2002) for various values of the parameters $\beta / a$ and $a / b$, where $b$ is the distance between the center of the particle and each of the plane walls. It was found that, for the case of impermeable walls under the situation of $\beta / a \rightarrow 0$, the particle mobility first decreases and then increases with increasing $a / b$. When the gaps between the particle and the plane walls turn thin, the particle can even migrate faster than it would as $a / b=0$ (by as much as $22 \%$ for an example of $a / b=0.99$ ). Exactly the same tendency of the dependence of $U / U_{0}$ on $a / b$ can be observed for the case that the two plane walls are prescribed with the far-field solute concentration profile under the situation of $\beta / a \gg 1$. This difference between the boundary effects on diffusiophoresis in a circular cylindrical pore and in a slit pore is striking, suggesting that the effect of viscous interactions is stronger or the effect of solutal

interactions is weaker in a circular pore than in a slit pore (and this fact can also be observed from the analytical method-ofreflection results for the two cases of pores). In general, the net boundary effect on diffusiophoresis of a particle is very much stronger in a circular pore than in a slit, which is understood knowing that a slit does not confine the particle from all directions (only from top and bottom) as a circular pore does.

\section{Notation}

$a$

A

$b$

$B_{n}, D_{n}$

C

$C_{0}$

$C_{\infty}$

$\mathbf{e}_{r}, \mathbf{e}_{z}$

$E_{\infty}$

G

$G_{n}^{-1 / 2}$

$I_{n}, K_{n}$

$k$

K

$L^{*}$

$P_{m}$

$r$

T

$T_{m}$

$\mathbf{U}, U$

$\mathbf{U}_{0}, U_{0}$

$\mathbf{v}$

$z$

Greek letters

$\alpha_{m}^{(1)}, \alpha_{m}^{(2)}, \alpha_{m}^{(3)}$

$\beta$

$\gamma_{i n}^{(j)}$

$\delta_{n}^{(1)}, \delta_{n}^{(2)}, \delta_{n}^{(3)}$

$\eta$ radius of the particle, $m$

diffusiophoretic mobility defined by Eq. (2b), $\mathrm{m}^{5} \mathrm{~s}^{-1}$

radius of the pore, $m$

coefficients in Eq. (23) or (24) for the flow field, $\mathrm{m}^{n+2} \mathrm{~s}^{-1}, \mathrm{~m}^{n} \mathrm{~s}^{-1}$

solute concentration field in the fluid, $\mathrm{m}^{-3}$

value of $C_{\infty}$ at the position of particle center, $\mathrm{m}^{-3}$

prescribed solute concentration field defined by Eq. (8), $\mathrm{m}^{-3}$

unit vectors in $r$ and $z$ directions

$=\left|\nabla C_{\infty}\right|, \mathrm{m}^{-4}$

dimensionless parameter defined by Eq. (31)

Gegenbauer polynomial of the first kind of order $n$ and degree $-1 / 2$

modified Bessel functions of the first and second kinds

Boltzmann's constant, $\mathrm{J} \mathrm{K}^{-1}$

Gibbs adsorption length defined by Eq. (6b), $\mathrm{m}$

characteristic length for the particle-solute interaction defined by Eq. (6c), m

Legendre polynomial of order $m$ radial spherical coordinate, $\mathrm{m}$

absolute temperature, $\mathrm{K}$

coefficients in Eqs. (12)-(14) for the solute concentration field, $\mathrm{m}^{m+2}, \mathrm{~m}^{-m+1}$

diffusiophoretic velocity of the particle, $\mathrm{m} \mathrm{s}^{-1}$

diffusiophoretic velocity of an isolated particle defined by Eq. (2), $\mathrm{m} \mathrm{s}^{-1}$

velocity field of the fluid, $\mathrm{m} \mathrm{s}^{-1}$

axial cylindrical coordinate, $\mathrm{m}$

functions of $r$ and $\theta$ defined by Eqs. (B.13)-(B.15), $\mathrm{m}^{-m-2}$

relaxation coefficient defined by Eq. (5), $\mathrm{m}$

functions of $r$ and $\theta$ defined by Eqs. (B.5) and (B.6), $\mathrm{m}^{-n-1}$ or $\mathrm{m}^{-n+1}$

functions of $r$ and $\theta$ defined by Eqs. (B.1)-(B.3), $\mathrm{m}^{-n-1}, \mathrm{~m}^{-n-2}, \mathrm{~m}^{-n-2}$ viscosity of the fluid, $\mathrm{kg} \mathrm{m}^{-1} \mathrm{~s}^{-1}$ 
$\theta, \phi \quad$ angular spherical coordinates

$\lambda \quad=a / b$

$\rho \quad$ radial cylindrical coordinate, $\mathrm{m}$

$\Psi \quad$ Stokes stream function for the fluid flow, $\mathrm{m}^{3} \mathrm{~s}^{-1}$

\section{Subscripts}

$\begin{array}{ll}p & \text { particle } \\ w & \text { wall }\end{array}$

\section{Acknowledgement}

This research was partly supported by the National Science Council of the Republic of China.

\section{Appendix A. Analysis of the diffusiophoresis of a spherical particle in a circular cylindrical pore by a method of reflec- tions}

In this appendix, we analyze the quasisteady diffusiophoretic motion of a colloidal sphere along the axis of a long circular cylindrical pore, as shown in Fig. 1, by a method of reflections. The effect of the pore wall on the diffusiophoretic velocity $\mathbf{U}$ of the particle is sought in expansions of $\lambda$, which equals $a / b$, the ratio of the particle-to-pore radii.

For the diffusiophoretic motion of a spherical particle in a pore with an impermeable wall, the governing equations (3) and (15) must be solved by satisfying the boundary conditions (4), (7), (8), and (18)-(20). The method-of-reflection solution consists of the following series, whose terms depend on increasing powers of $\lambda$ :

$C=C_{0}+E_{\infty} z+C_{p}^{(1)}+C_{w}^{(1)}+C_{p}^{(2)}+C_{w}^{(2)}+\cdots$,

$\mathbf{v}=\mathbf{v}_{p}^{(1)}+\mathbf{v}_{w}^{(1)}+\mathbf{v}_{p}^{(2)}+\mathbf{v}_{w}^{(2)}+\cdots$,

where subscripts $p$ and $w$ represent the reflections from particle and wall, respectively, and the superscript $(i)$ denotes the $i$ th reflection from that surface. In these series, all the expansion sets of the corresponding concentration and velocity fields for the fluid solution must satisfy Eqs. (3) and (15). The advantage of this method is that it is necessary to consider boundary conditions associated with only one surface at a time.

According to Eq. (A.1), the velocity of the particle can also be expressed in the series form

$\mathbf{U}=\left(U_{0}+v_{\infty}\right) \mathbf{e}_{z}+\mathbf{U}^{(1)}+\mathbf{U}^{(2)}+\cdots$.

In these expressions, $U_{0}=A E_{\infty}$ is the diffusiophoretic velocity of an identical particle suspended freely in the continuous phase far from the wall given by Eq. (2); $v_{\infty}$ is the diffusioosmotic velocity of the fluid far from the particle caused by the existence of the pore wall defined in Eq. $(20) ; \mathbf{U}^{(i)}$ is related to $\nabla C_{w}^{(i)}$ and $\mathbf{v}_{w}^{(i)}$ by (Keh and Luo, 1995)

$\mathbf{U}^{(i)}=A\left[\nabla C_{w}^{(i)}\right]_{0}+\left[\mathbf{v}_{w}^{(i)}\right]_{0}+\frac{a^{2}}{6}\left[\nabla^{2} \mathbf{v}_{w}^{(i)}\right]_{0}$,

where the subscript 0 to variables inside brackets denotes evaluation at the position of the particle center.

Th solution for the first reflected fields from the particle is

$C_{p}^{(1)}=G E_{\infty} a^{3} r^{-2} \cos \theta$,

$\mathbf{v}_{p}^{(1)}=\frac{1}{2} U_{0} a^{3} r^{-3}\left(2 \cos \theta \mathbf{e}_{r}+\sin \theta \mathbf{e}_{\theta}\right)$,

where $G$ was defined by Eq. (31). The velocity distribution shown in Eq. (A.4b) is identical to the irrotational flow surrounding a rigid sphere moving with velocity $U_{0} \mathbf{e}_{z}$.

The boundary conditions for the $i$ th reflected fields from the wall are derived from Eqs. (7), (8), (19), and (20),

$\rho=b: \quad \frac{\partial C_{w}^{(i)}}{\partial \rho}=-\frac{\partial C_{p}^{(i)}}{\partial \rho}$,

$\mathbf{v}_{w}^{(i)}=-\mathbf{v}_{p}^{(i)}-\left[\frac{k T}{\eta} L_{w}^{*} K_{w} \frac{\partial C_{w}^{(i)}}{\partial z}+v_{\infty}\right] \mathbf{e}_{z}$

$|z| \rightarrow \infty: \quad C_{w}^{(i)} \rightarrow 0$

$\mathbf{v}_{w}^{(i)} \rightarrow \mathbf{0}$.

The solution of $C_{w}^{(1)}$ is obtained by applying Fourier sine transform on variable $z$ in Eqs. (3) and (A.5a), (A.5c), with the result

$C_{w}^{(1)}=\frac{2}{\pi} G E_{\infty} a \lambda^{2} \int_{0}^{\infty} \omega \frac{K_{1}(\omega)}{I_{1}(\omega)} I_{0}\left(\frac{\rho}{b} \omega\right) \sin \left(\frac{z}{b} \omega\right) \mathrm{d} \omega$,

(A.6a)

where $I_{n}(\omega)$ and $K_{n}(\omega)$ are modified Bessel functions of the first kind and second kind, respectively, of order $n . \mathbf{v}_{w}^{(1)}$ can be solved by applying Fourier cosine transforms twice to the Stokes equation (15) and boundary conditions (A.5b), (A.5d), which results in

$$
\begin{aligned}
\mathbf{v}_{w}^{(1)}= & \frac{U_{0} \lambda^{3}}{\pi} \int_{0}^{\infty}\left\{\left[2 A_{1}(\omega) I_{0}\left(\frac{\rho}{b} \omega\right)+A_{1}(\omega) \frac{\rho}{b} \omega I_{1}\left(\frac{\rho}{b} \omega\right)\right.\right. \\
& \left.+A_{2}(\omega) I_{0}\left(\frac{\rho}{b} \omega\right)\right] \cos \left(\frac{z}{b} \omega\right) \mathbf{e}_{z}+\left[A_{1}(\omega) \frac{\rho}{b} \omega\right. \\
& \left.\left.\times I_{0}\left(\frac{\rho}{b} \omega\right)+A_{2}(\omega) I_{1}\left(\frac{\rho}{b} \omega\right)\right] \sin \left(\frac{z}{b} \omega\right) \mathbf{e}_{\rho}\right\} \mathrm{d} \omega,
\end{aligned}
$$

where

$$
A_{1}(\omega)=\frac{1-2 \alpha G(1+\beta / a)}{\left[I_{1}(\omega)\right]^{2}-I_{0}(\omega) I_{2}(\omega)}
$$

$$
A_{2}(\omega)=\frac{-\omega^{2}\left[I_{1}(\omega) K_{1}(\omega)+I_{0}(\omega) K_{2}(\omega)\right]+2 \alpha G(1+\beta / a) \omega I_{0}(\omega)\left[I_{1}(\omega)\right]^{-1}}{\left[I_{1}(\omega)\right]^{2}-I_{0}(\omega) I_{2}(\omega)}
$$


The contributions of $C_{w}^{(1)}$ and $\mathbf{v}_{w}^{(1)}$ to the particle velocity are determined by using Eq. (A.3),

$$
\begin{aligned}
\mathbf{U}_{s}^{(1)}= & A\left[\nabla C_{w}^{(1)}\right]_{r=0}=d_{1} G \lambda^{3} U_{0} \mathbf{e}_{z}, \\
\mathbf{U}_{h}^{(1)}= & {\left[\mathbf{v}_{w}^{(1)}+\frac{a^{2}}{6} \nabla^{2} \mathbf{v}_{w}^{(1)}\right]_{r=0}=\left\{\left[d_{2}+d_{4} \alpha G\left(1+\frac{\beta}{a}\right)\right] \lambda^{3}\right.} \\
& \left.+d_{3}\left[1-2 \alpha G\left(1+\frac{\beta}{a}\right)\right] \lambda^{5}\right\} U_{0} \mathbf{e}_{z}, \\
\mathbf{U}^{(1)}= & \mathbf{U}_{s}^{(1)}+\mathbf{U}_{h}^{(1)}=\left\{\left[d_{1} G+d_{2}+d_{4} \alpha G\left(1+\frac{\beta}{a}\right)\right] \lambda^{3}\right. \\
& \left.+d_{3}\left[1-2 \alpha G\left(1+\frac{\beta}{a}\right)\right] \lambda^{5}\right\} U_{0} \mathbf{e}_{z},
\end{aligned}
$$

where

$$
\begin{aligned}
d_{1} & =\frac{2}{\pi} \int_{0}^{\infty} \omega^{2} \frac{K_{1}(\omega)}{I_{1}(\omega)} \mathrm{d} \omega=1.59365, \\
d_{2} & =\frac{1}{\pi} \int_{0}^{\infty} \frac{2-\omega^{2}\left[I_{1}(\omega) K_{1}(\omega)+I_{0}(\omega) K_{2}(\omega)\right]}{\left[I_{1}(\omega)\right]^{2}-I_{0}(\omega) I_{2}(\omega)} \\
& =-2.08669,
\end{aligned}
$$

$$
d_{3}=\frac{1}{3 \pi} \int_{0}^{\infty} \frac{\omega^{2}}{\left[I_{1}(\omega)\right]^{2}-I_{0}(\omega) I_{2}(\omega)} \mathrm{d} \omega=1.89632,
$$

$$
d_{4}=\frac{2}{\pi} \int_{0}^{\infty} \frac{\omega I_{0}(\omega)\left[I_{1}(\omega)\right]^{-1}-2}{\left[I_{1}(\omega)\right]^{2}-I_{0}(\omega) I_{2}(\omega)} \mathrm{d} \omega=2.57974
$$

Eq. (A.8a) shows that the reflected concentration field from the impermeable wall can increase (if $G>0$ or $\beta / a<1 / 2$ ) or decrease (if $G<0$ or $\beta / a>1 / 2$ ) the velocity of the diffusiophoretic particle, while Eq. (A.8b) indicates that the reflected velocity field is to decrease this velocity for the case of $\alpha=0$ (where the parameter $\alpha$ is defined right after Eq. (28)); the net effect of the reflected fields is expressed by Eq. (A.8c), which can enhance or retard the movement of the particle, depending on the combination of the values of $G$ (or $\beta / a$ ) and $\lambda$. When $G=0$ (or $\beta / a=1 / 2$ ), the reflected solute concentration field makes no contribution to the diffusiophoretic velocity. Eq. (A.8c) indicates that the wall correction to the diffusiophoretic velocity of the particle is $\mathrm{O}\left(\lambda^{3}\right)$, which is weaker than that obtained for the corresponding sedimentation problem, in which the leading boundary effect is $\mathrm{O}(\lambda)$. Note that, for the case of $\alpha=0$, the necessary condition for the wall enhancement on the diffusiophoretic motion to occur is a small value of $\beta / a$ and a value of $\lambda$ close to unity such that the relation $d_{3} \lambda^{5}>-\left(d_{2}+d_{1} G\right) \lambda^{3}$ is warranted.

The solution for the second reflected fields from the particle is

$$
\begin{aligned}
C_{p}^{(2)}= & E_{\infty}\left[d_{1} G^{2} \lambda^{3} a^{3} r^{-2} \cos \theta+\mathrm{O}\left(\lambda^{5} a^{5}\right)\right], \\
\mathbf{v}_{p}^{(2)}= & \frac{1}{2} U_{0}\left[d_{1} G \lambda^{3} a^{3} r^{-3}\left(2 \cos \theta \mathbf{e}_{r}+\sin \theta \mathbf{e}_{\theta}\right)\right. \\
& \left.+\mathrm{O}\left(\lambda^{5} a^{3}\right)\right] .
\end{aligned}
$$

The boundary conditions for the second reflected fields from the wall are obtained by substituting the results of $C_{p}^{(2)}$ and $\mathbf{v}_{p}^{(2)}$ into Eq. (A.5), with which Eqs. (3) and (15) can be solved as before to yield

$$
\begin{aligned}
& {\left[\nabla C_{w}^{(2)}\right]_{r=0}=d_{1}^{2} G^{2} \lambda^{6} E_{\infty},} \\
& {\left[\mathbf{v}_{w}^{(2)}+\frac{a^{2}}{6} \nabla^{2} \mathbf{v}_{w}^{(2)}\right]_{r=0}} \\
& =d_{1} G\left\{\left[d_{2}+d_{4} \alpha G\left(1+\frac{\beta}{a}\right)\right] \lambda^{6}\right. \\
& \left.\quad+d_{3}\left[1-2 \alpha G\left(1+\frac{\beta}{a}\right)\right] \lambda^{8}\right\} U_{0} \mathbf{e}_{z} .
\end{aligned}
$$

The contribution of the second reflected fields to the velocity of the particle is obtained by using Eqs. (A.3) and (A.11), which gives

$$
\begin{aligned}
\mathbf{U}^{(2)}= & d_{1} G\left\{\left[d_{1} G+d_{2}+d_{4} \alpha G\left(1+\frac{\beta}{a}\right)\right] \lambda^{6}\right. \\
& \left.+d_{3}\left[1-2 \alpha G\left(1+\frac{\beta}{a}\right)\right] \lambda^{8}\right\} U_{0} \mathbf{e}_{z} .
\end{aligned}
$$

Obviously, $\mathbf{U}^{(3)}$ will be $\mathrm{O}\left(\lambda^{9}\right)$. With the substitution of Eqs. (A.8c) and (A.12) into Eq. (A.2), the particle velocity can be expressed as $\mathbf{U}=U \mathbf{e}_{z}$ with

$$
\begin{aligned}
U= & \left\{\left[1-\alpha\left(1+\frac{\beta}{a}\right)\right]+\left[d_{1} G+d_{2}+d_{4} \alpha G\left(1+\frac{\beta}{a}\right)\right] \lambda^{3}\right. \\
& +d_{3}\left[1-2 \alpha G\left(1+\frac{\beta}{a}\right)\right] \lambda^{5}+d_{1} G\left[d_{1} G+d_{2}\right. \\
& \left.+d_{4} \alpha G\left(1+\frac{\beta}{a}\right)\right] \lambda^{6} \\
& \left.+d_{1} d_{3} G\left[1-2 \alpha G\left(1+\frac{\beta}{a}\right)\right] \lambda^{8}\right\} U_{0} .
\end{aligned}
$$

In the limit $\beta / a=0$ (no polarization of the diffuse layer surrounding the particle), $G=1 / 2$ and the above equation reduces to

$$
\begin{aligned}
U= & {\left[1+\frac{1}{2}\left(d_{1}+2 d_{2}+d_{4} \alpha\right) \lambda^{3}+d_{3}(1-\alpha) \lambda^{5}\right.} \\
& +\frac{1}{4} d_{1}\left(d_{1}+2 d_{2}+d_{4} \alpha\right) \lambda^{6}+\frac{1}{2} d_{1} d_{3}(1-\alpha) \lambda^{8} \\
& \left.+\mathrm{O}\left(\lambda^{9}\right)\right] U_{0} .
\end{aligned}
$$

This formula can also be used to express the corresponding electrophoretic velocity of a dielectric sphere with $U_{0}$ representing the value of this velocity when the pore does not exist (given by the well-known Smoluchowski equation), and is consistent with the result of an earlier analysis for the wall effect on electrophoresis of a charged sphere with a thin unpolarized double layer correct to $\mathrm{O}\left(\lambda^{6}\right)$ (Keh and Anderson, 1985).

For the case that a linear solute concentration profile is prescribed on the pore wall which is consistent with the far-field distribution, namely, the boundary condition (7) is replaced by Eq. (9), the series expansions (A.1) and (A.2), the solutions of $C_{p}^{(1)}$ and $\mathbf{v}_{p}^{(1)}$ in Eq. (A.4), and the boundary conditions for $C_{w}^{(i)}$ and $\mathbf{v}_{w}^{(i)}$ in Eqs. (A.5b)-(A.5d) are still valid, while Eq. (A.5a) 
becomes

$\rho=b: \quad C_{w}^{(i)}=-C_{p}^{(i)}$.

The solution of $C_{w}^{(1)}$ satisfying Eqs. (3), (A.15), and (A.5c) is $C_{w}^{(1)}=\frac{2}{\pi} G E_{\infty} a \lambda^{2} \int_{0}^{\infty} \omega \frac{K_{0}(\omega)}{I_{0}(\omega)} I_{0}\left(\frac{\rho}{b} \omega\right) \sin \left(\frac{z}{b} \omega\right) \mathrm{d} \omega$

while the solution of $\mathbf{v}_{w}^{(1)}$ is unchanged from Eq. (A.6b). The results of the following reflected fields and of the particle velocity are also obtained from Eqs. (A.8)-(A.14) by replacing $d_{1}$ by $\bar{d}_{1}$, where

$\bar{d}_{1}=-\frac{2}{\pi} \int_{0}^{\infty} \omega^{2} \frac{K_{0}(\omega)}{I_{0}(\omega)} \mathrm{d} \omega=-0.411822$.

Thus, contrary to the effect of an impermeable pore wall, the reflected solute concentration field from a pore wall with the prescribed far-field concentration distribution reduces the velocity of the particle if $G>0$ or $\beta / a<1 / 2$ and enhances this velocity if $G<0$ or $\beta / a>1 / 2$. When $G=0$ or $\beta / a=1 / 2$, the two types of plane wall will produce the same effects on the diffusiophoretic motion of the particle. Under the condition that the values of $\beta / a$ and $\lambda$ are sufficiently large such that $d_{3} \lambda^{5}>-\left(d_{2}+\bar{d}_{1} G\right) \lambda^{3}$, the net effect of a pore wall prescribed with the far-field concentration distribution can enhance the diffusiophoretic migration of a particle for the case of $\alpha=0$.

\section{Appendix B. Definitions of some functions in Section 2}

The functions $\delta_{m}^{(1)}, \delta_{m}^{(2)}$, and $\delta_{m}^{(3)}$ in Eqs. (13) and (14) are defined by

$$
\begin{aligned}
\delta_{m}^{(1)}(r, \theta)= & \int_{0}^{\infty} H(\omega) I_{0}(\omega r \sin \theta) \sin (\omega r \cos \theta) \mathrm{d} \omega \\
& +r^{-m-1} P_{m}(\cos \theta), \\
\delta_{m}^{(2)}(r, \theta)= & \int_{0}^{\infty} \omega H(\omega)\left[I_{0}(\omega r \sin \theta) \cos (\omega r \cos \theta) \cos \theta\right. \\
& \left.+I_{1}(\omega r \sin \theta) \sin (\omega r \cos \theta) \sin \theta\right] \mathrm{d} \omega \\
& -(m+1) r^{-m-2} P_{m}(\cos \theta), \\
\delta_{m}^{(3)}(r, \theta)= & \int_{0}^{\infty} \omega H(\omega)\left\{I_{1}(\omega r \sin \theta)\right. \\
& \times \sin \theta[2 \omega r \cos (\omega r \cos \theta) \cos \theta \\
& +\sin (\omega r \cos \theta)]+I_{0}(\omega r \sin \theta) \\
& \times[2 \cos (\omega r \cos \theta) \cos \theta \\
& -\omega r \sin (\omega r \cos \theta) \cos (2 \theta)]\} \mathrm{d} \omega \\
& +m(m+1) r^{-m-2} P_{m}(\cos \theta),
\end{aligned}
$$

where

$H(\omega)=(-1)^{(m-2 v+1) / 2} \frac{2 \omega^{m}}{\pi m !} \frac{K_{v}(b \omega)}{I_{v}(b \omega)} ;$

$I_{v}$ and $K_{v}$ are the modified Bessel functions of the first and second kinds, respectively, of order $v ; v=1$ if Eq. (7) is used for the boundary condition of the solute concentration field at the pore wall and $v=0$ if Eq. (9) is used.
The functions $\gamma_{i n}^{(j)}$ for $i$ and $j$ equal to 1 or 2 in Eqs. (24) and (25) are defined by

$$
\begin{aligned}
\gamma_{i n}^{(1)}(r, \theta)= & \int_{0}^{\infty}\left[S_{i}^{(1)}(\omega) I_{0}(\omega r \sin \theta) r \sin \theta\right. \\
& \left.+S_{i}^{(2)}(\omega) I_{1}(\omega r \sin \theta)\right] \omega \sin (\omega r \cos \theta) \mathrm{d} \omega \\
& -r^{-n+2 i-3}\left[(n+1) G_{n+1}^{-1 / 2}(\cos \theta) \csc \theta\right. \\
& \left.-2(i-1) G_{n}^{-1 / 2}(\cos \theta) \cot \theta\right], \\
\gamma_{i n}^{(2)}(r, \theta)= & \int_{0}^{\infty}\left\{S _ { i } ^ { ( 1 ) } ( \omega ) \left[2 I_{0}(\omega r \sin \theta)\right.\right. \\
& \left.\left.+I_{1}(\omega r \sin \theta) \omega r \sin \theta\right]+\omega S_{i}^{(2)}(\omega) I_{0}(\omega r \sin \theta)\right\} \\
& \times \cos (\omega r \cos \theta) \mathrm{d} \omega-r^{-n+2 i-3}\left[P_{n}(\cos \theta)\right. \\
& \left.+2(i-1) G_{n}^{-1 / 2}(\cos \theta)\right] .
\end{aligned}
$$

In Eqs. (B.5) and (B.6),

$S_{i}^{1}(\omega)=\frac{A_{i n}^{(1)}(\omega) I_{0}(b \omega)-A_{i n}^{(2)}(\omega) I_{1}(b \omega)}{b \omega I_{0}^{2}(b \omega)-2 I_{0}(b \omega) I_{1}(b \omega)-b \omega I_{1}^{2}(b \omega)}$,

$S_{i}^{(2)}(\omega)=\frac{A_{i n}^{(1)}(\omega)-b \omega I_{0}(b \omega) S_{i}^{(1)}(\omega)}{\omega I_{1}(b \omega)}$,

where

$A_{1 n}^{(1)}(\omega)=-(-1)^{n / 2} \frac{2 \omega^{n}}{\pi n !} K_{1}(b \omega)$,

$A_{2 n}^{(1)}(\omega)=(-1)^{n / 2} \frac{2 \omega^{n-2}}{\pi n !}\left[(n-2)(n-3) K_{1}(b \omega)\right.$

$\left.-(2 n-3) b \omega K_{0}(b \omega)\right]$,

$A_{1 n}^{(2)}(\omega)=(-1)^{n / 2} \frac{2 \omega^{n}}{\pi n !} K_{0}(b \omega)$,

$A_{2 n}^{(2)}(\omega)=(-1)^{n / 2} \frac{2 \omega^{n-2}}{\pi n !}\left[(2 n-3) b \omega K_{1}(b \omega)\right.$

$$
\left.-n(n-1) K_{0}(b \omega)\right] \text {. }
$$

The functions $\alpha_{m}^{(i)}$ for $i=1,2$, and 3 in Eq. (25) are defined by

$$
\begin{aligned}
\alpha_{m}^{(1)}(r, \theta)= & \int_{0}^{\infty} B_{m}(\omega)\left[\omega r \sin \theta I_{0}(\omega r \sin \theta)\right. \\
& \left.-b \omega \frac{I_{0}(b \omega)}{I_{1}(b \omega)} I_{1}(\omega r \sin \theta)\right] \sin (\omega r \cos \theta) \mathrm{d} \omega
\end{aligned}
$$

$$
\begin{aligned}
\alpha_{m}^{(2)}(r, \theta)= & \int_{0}^{\infty} B_{m}(\omega)\left[\left(2-b \omega \frac{I_{0}(b \omega)}{I_{1}(b \omega)}\right) I_{0}(\omega r \sin \theta)\right. \\
& \left.+\omega r \sin \theta I_{1}(\omega r \sin \theta)\right] \cos (\omega r \cos \theta) \mathrm{d} \omega,
\end{aligned}
$$

$$
\begin{aligned}
\alpha_{m}^{(3)}(r, \theta)= & \int_{0}^{\infty} \omega H(\omega)\left[-I_{0}(\omega r \sin \theta) \cos (\omega r \cos \theta) \sin \theta\right. \\
& \left.+I_{1}(\omega r \sin \theta) \sin (\omega r \cos \theta) \cos \theta\right] \mathrm{d} \omega \\
& +\frac{m}{\sin \theta} r^{-m-2}\left[\cos \theta P_{m}(\cos \theta)\right. \\
& \left.-P_{m-1}(\cos \theta)\right]
\end{aligned}
$$


where

$$
\begin{aligned}
B_{m}(\omega)= & (-1)^{(m-1) / 2} \frac{2 \omega^{m+1}}{\pi m !} \\
& \times \frac{K_{0}(b \omega) I_{1}(b \omega)+K_{1}(b \omega) I_{0}(b \omega)}{b \omega I_{0}^{2}(b \omega)-2 I_{0}(b \omega) I_{1}(b \omega)-b \omega I_{1}^{2}(b \omega)} .
\end{aligned}
$$

\section{References}

Anderson, J.L., 1986. Transport mechanisms of biological colloids. Annals of the New York Academy of Sciences Biochemical Engineering IV 469, $166-177$.

Anderson, J.L., 1989. Colloid transport by interfacial forces. Annual Review of Fluid Mechanics 21, 61-99.

Anderson, J.L., Prieve, D.C., 1991. Diffusiophoresis caused by gradients of strongly adsorbing solutes. Langmuir 7, 403-406.

Anderson, J.L., Lowell, M.E., Prieve, D.C., 1982. Motion of a particle generated by chemical gradients. Part 1. Non-electrolytes. Journal of Fluid Mechanics 117, 107-121.

Chen, P.Y., Keh, H.J., 2002. Diffusiophoresis of a colloidal sphere in nonelectrolyte gradients parallel to one or two plane walls. Chemical Engineering Science 57, 2885-2899.

Chen, S.B., Keh, H.J., 1999. In: Hsu, J. (Ed.), Interfacial Forces and Fields. Dekker, New York.
Dukhin, S.S., Derjaguin, B.V., 1974. Electrokinetic phenomena. In: Matijevic, E. (Ed.), Surface and Colloid Science, vol. 7. Wiley, New York.

Ebel, J.P., Anderson, J.L., Prieve, D.C., 1988. Diffusiophoresis of latex particles in electrolyte gradients. Langmuir 4, 396-406.

Happel, J., Brenner, H., 1983. Low Reynolds Number Hydrodynamics. Nijhoff, Dordrecht, The Netherlands.

Keh, H.J., Anderson, J.L., 1985. Boundary effects on electrophoretic motion of colloidal spheres. Journal of Fluid Mechanics 183, 458-475.

Keh, H.J., Chiou, S.B., 1996. Electrophoresis of a colloidal sphere in a circular cylindrical pore. A.I.Ch.E. Journal 42, 1397-1406.

Keh, H.J., Jan, J.S., 1996. Boundary effects on diffusiophoresis and electrophoresis: motion of a colloidal sphere normal to a plane wall. Journal of Colloid and Interface Science 183, 458-475.

Keh, H.J., Luo, S.C., 1995. Particle interactions in diffusiophoresis in nonelectrolyte gradients. Physics of Fluids 7, 2122-2131.

Leichtberg, S., Pfeffer, R., Weinbaum, S., 1976. Stokes flow past finite coaxial clusters of spheres in a circular cylinder. International Journal of Multiphase Flow 3, 147-169.

O'Brien, R.W., 1983. The solution of the electrokinetic equations for colloidal particles with thin double layers. Journal of Colloid and Interface Science 92, 204-216.

Staffeld, P.O., Quinn, J.A., 1989. Diffusion-induced banding of colloid particles via diffusiophoresis. 2. Non-electrolytes. Journal of Colloid and Interface Science 130, 88-100. 